\title{
Role of MR arthrography in assessment of glenohumeral instability
}

\section{Hamdan Abdelhameed Alya ${ }^{a}$ Mohammad Hassan Alkousy ${ }^{\mathrm{a}}$, Khaled Fawzy Zaky ${ }^{\mathrm{a}}$, Mohammad Tharwat Mahmoud Solymana.}

\author{
aDepartment of Radio-diagnosis, Faculty of Medicine, Sohag University, Sohag, Egypt.
}

\begin{abstract}
Background: The glenohumeral joint has a unique structure which allows for the widest range of motion among all joints in the human body; however this unfortunately makes the joint inherently unstable. Various imaging techniques are available for assessment of shoulder joint abnormalities. Magnetic resonance arthrography is considered the best modality for the assessment of disorders related to shoulder instability. The injection of para magnetic contrast solution into the joint cavity induced distension of the capsule allowing separation of the intra articular structures and beret visualization of shoulder disorders. However, arthrography is a time-consuming, minimally invasive procedure, which, although generally safe, it might carries some risks like hemorrhage and infection.
\end{abstract}

Objectives: The aim of this work was to evaluate the diagnostic performance of MR arthrography (MRA) in the detection of shoulder disorders in patients having shoulder instability.

Patients and methods: Between February 2017 and March 2021, 50 patients with a clinical diagnosis of shoulder instability were examined by MR arthrography. MR arthrographic finings including the different labral tears, rotator-cuff tears (RCTs) and Hill-Sachs lesions were recognized and compared to surgical arthroscope ' the gold standard'. Sensitivity, specificity, accuracy, PPV and NPV were calculated for MR arthrography.

Results: MRA showed sensitivity and specificity of about $90 \%$ for the detection of classic Bankart lesions, sensitivity of 91.8 and specificity of $100 \%$, for detecting bony Bankart lesions, sensitivity and specificity of $91.6 \%$, and $100 \%$ for the detection of SLAP lesions. The sensitivity and specificity for the detection of HillSachs were $92.31 \%, 100 \%$ respectively; the sensitivity and specificity in the detection of RCTs were $95.24 \%$ and $100 \%$. Conclusion: MR arthrography has a high diagnostic performance in the detection of different labral tears. RCTs (in particular partial thickness RCTs) and Hill-Sachs lesions.

Keywords: MR arthrogram - Arthroscope - Rotator cuff - Tears - Hill-Sach's lesions.

\section{Introduction}

The shoulder joint enjoys the widest range of motion of all the joints in the human body; therefore it requires a delicate balance between stability and mobility (Chang and Polster, 2015). This depends on a complex interaction between soft tissues and bony articulation to allow for such wide range of motion (ROM) and minimize instability (Degen et al., 2013)

To ensure stability, the shoulder is stabilized by both static and dynamic stabilizers. The static stabilizers include the bony configurations of the

Copyright: () Aly et al. (2021) Immediate open access to its content on the principle that making 
glenoid and the humerus, the glenoid labrum, the joint capsule, and the glenohumeral ligaments. The dynamic stabilizers include rotator cuff muscles and, to a lesser degree, the long head of the biceps and the deltoid muscle (Abboud and Soslowsky, 2002).

Direct MR arthrography is the imaging modality of choice for the diagnosis of shoulder instability and labral lesions (Omoumi et al., 2010). MR arthrography can be performed directly with intra-articular contrast material injection or indirectly with intravenous contrast material injection (Chang et al., 2008)

Direct MR arthrography refers precisely to the direct administration of dilute gadolinium solution into a joint, followed by MRI imaging. The overwhelming advantage of direct MR arthrography lies in the benefit gained from joint distension which allows the redundant capsule to be distinguished from the adjacent labrum and also allows for the passage of contrast material into the substance of the labrum in the presence of unstable tears, as well as between the labrum and the glenoid in case of labral detachment. These two patterns represent common types of labral pathologic abnormalities that may otherwise go unnoticed. The ability of direct arthrography to depict these patterns offers a clear diagnostic advantage in comparison to conventional MR imaging, particularly in patients with chronic symptoms at presentation or those without a history of acute posttraumatic injury. Furthermore, gadolinium causes T1 shortening, resulting in high signal intensity fluid on T1-weighted images. On applying fat-saturation to the T1-weighted sequences, the signal from fat is nulled and the exact distribution of contrast solution can be seen more easily. The fluid also remains high-signal intensity on T2-weighted images (Chundru et al.,
2009) The aim of the study was to assess the diagnostic performance of 1.5Tesla MR arthrography compared with surgical arthroscope for the assessment of abnormalities of the labrum, rotator cuff, and glenohumeral joint.

\section{Patients and methods}

This was a retrospective study between February 2017 and March 2021conducted on 50 patients suspected clinically to have shoulder instability who subsequently underwent arthroscopy. The age range of the 50 patients was 18-50 years (mean age, 34 years). All patients underwent arthroscopy within 1 month of the MR arthrography examination (range, 1-30 days; mean time between MR arthrography and arthroscope, 11 days). Findings on MR arthrography were then reported and compared to arthroscopy 'Gold standard'.

Setting: Department of radio-diagnosis, Sohag University Hospital, Sohag, Egypt.

The exclusion criteria: were fractures at the shoulder and infection. Also all metallic body implants (that such as cardiac pacemakers, clips for brain aneurysm, cochlear implants etc.) were considered absolute contraindications to the performance of MR arthrography.

For performing MRA, US guided injection of 12-20 $\mathrm{cc}$ of dilute gadolinium solution (approximately 1:200 dilution) into the shoulder joint via an anterior 'rotator interval' and posterior approaches was done using a 22-gauge spinal needle. Subsequent MRI sequences included coronal T1WIs with fat suppression, coronal PD with fat suppression, sagittal T1-weighted with fat suppression, and axial T1WIs with fat-saturated sequences. A 1.5-T scanner was used. All MR arthrography images were obtained with a $12-\mathrm{cm}$ 
FOV and a 4-mm slice thickness (with a $10 \%$ inter slice gap).

Shoulder arthroscopy was performed by a single expert orthopedic surgeon specialized in shoulder surgery using a 4-mm arthroscope placed through a small portal. Arthroscopy was done under general anesthesia. The patient was in the beach chair position with the arm affected being placed at the side of the body, the elbow flexed and the forearm resting on an arm rest, with neutral rotation.

Depending on the type of lesion identified, tears found were either débrided or repaired. All compartments of the affected shoulder were examined in order to detect various shoulder abnormalities.

After finishing surgery, the findings were incorporated on a standard form by the operator. The results on MRI were then compared with the results of arthroscopy.

\section{Ethical approval}

The study protocol was approved by the Ethical Committee of Faculty of Medicine, Sohag University, and written informed consent was obtained from all patients.

\section{Statistical analysis}

Data was analyzed using STATA version 14.2 (Stata Statistical Software: Release 14.2 College Station, TX: StataCorp LP.).
Quantitative data was represented as mean, standard deviation, median and range. Qualitative data was presented as number and percentage. The sensitivity, specificity, and positive and negative likelihood ratios were calculated for MRA versus arthroscopy. Graphs were produced by using Excel or STATA program. P value was considered significant if it was less than 0.05 .

\section{Results}

This study included 50 patients; 47 males, 3 females (mean age 34 years), suffering from symptoms of glenohumeral instability in the form of dislocation whether traumatic or non traumatic associated with pain and/or limitation of movement. All of the 50 patients were subjected to MR arthrography. Arthroscopy was done for all patients. The arthroscopic results were then compared with the collected data of imaging.

Arthroscopic examination, confirmed pathological lesions in 46 out of 50 patients included in this study. The findings include 19 classic Bankart lesion, 3 Perthes lesions, 5 ALPSA 2 GLAD, 12 SLAP, 27 humeral head (Hill-Sachs) fractures and 7 Bony Bankart's lesions (Table 1).The sensitivity, specificity, positive predictive and negative predictive values as well as accuracy of MR arthrography were compared to arthroscopic findings ' the gold standard' (Table 2). 
Table 1: The 5 main arthroscopic findings:

\begin{tabular}{|l||l||}
\hline \multicolumn{1}{|c|}{ Type of findings } & \multicolumn{1}{|c|}{ Number (\%) } \\
\hline \hline Bankart's lesions & $30(60.00 \%)$. \\
- Classic Bankart's & 10 \\
- Bony Bankart's & 11 \\
- Perthes lesion & 2 \\
- ALPSA & 5 \\
- GLAD lesion & 2 \\
\hline \hline SLAP lesions & \\
\hline \hline Hill Sachs fractures & $12(24.00 \%)$ \\
\hline \hline Full thickness RCT & $26(52.00 \%)$ \\
\hline \hline Partial thickness RCT & $1(2.00 \%)$ \\
\hline
\end{tabular}

(ALPSA, anterior labrum periosteal sleeve avulsion; GLAD, glenoid labrum articular disruption; SLAP, superior labrum anterior - posterior; RCT, rotator cuff tear).

Table 2. Comparative study of MR arthrography with arthroscopic correlation:

\begin{tabular}{|lccccc|}
\hline MR arthrography findings & & & & & \\
& Sensitivity (\%) & Specificity (\%) & PPV (\%) & NPV (\%) & Accuracy \\
\hline Classic Bankart's lesion & 90 & 90 & 69.23 & 97.3 & 85 \\
Perthes lesion & 66.6 & 100 & 100 & 96.7 & 97.8 \\
ALPSA & 100 & 97.5 & 97.5 & 100 & 97.8 \\
GLAD & 60 & 97.6 & 75 & 95.4 & 95.7 \\
SLAP & 91.67 & 100 & 97.44 & 95.38 & 0.92 \\
Bony Bankart's & 91.82 & 100 & 100 & 95.12 & 90.91 \\
Hill Sachs fracture & 92.3 & 100 & 100 & 92.31 & 96.15 \\
RCT & 100 & 95.24 & 80 & 100 & 97.62 \\
Partial thickness RCT & 77.78 & 100 & 100 & 95.35 & 88.89 \\
& & & & & \\
\hline
\end{tabular}

(ALPSA, anterior labrum periosteal sleeve avulsion; GLAD, glenoid labrum articular disruption; SLAP, superior labrum anterior - posterior; RCT, rotator cuff tear).

\section{Discussion}

Owing to its unique structure, the shoulder joint enjoys the widest range of motion among all joints of the human body; however, this also makes it particularly at risk of instability. Anterior instability is the most common form, accounting for about $95 \%$ of all GHJ instability events and typically seen among young, athletic populations 
especially during contact sports with the shoulder in the abducted and externally rotated position. The remaining $5 \%$ have posterior instability (3\%), inferior, superior or multidirectional instability (Burke et al., 2020) .

Although conventional MR imaging allows direct visualization of major anatomic structures of the shoulder, smaller intra articular structures, such as the labrum, glenohumeral ligaments, and articular surface of the rotator cuff tendon can be hard to evaluate in the absence of a joint effusion. MR arthrography is regarded as the gold-standard imaging modality for shoulder instability. Indeed, the injection of paramagnetic contrast agent into the joint allows for capsule distension and better visualization of intra articular structures

(Sconfienza et al., 2018) .

For performing direct MRA, a mix of diluted para magnetic contrast agent was used. The delivery of contrast into the joint was performed under sonographic guidance using both anterior 'rotator interval' and posterior approaches.

A 20 - or 22-gauge 3.5 -inch $(0.8 \times 50-\mathrm{mm}$ beveled) spinal needle was used to puncture the glenohumeral joint. US-guided procedure has the advantage of not exposing the patients or the radiologist to ionizing radiation when compared to Fluoroscopy-guided procedure and is also relatively simple and causes no patient discomfort as well as less time consuming (Gokalp et al., 2010)

In the current study, the mean volume of the mixture of the contrast agent was about $19 \mathrm{ml}$ (range of 10-24 ml). MR arrhography is usually done with a maximum required volume of $15 \mathrm{ml}$ for rotator cuff lesions and $20 \mathrm{ml}$ for instability.
For optimal visualization of normal and abnormal anatomical structures, we stopped injection of the contrast mixture only when the distention became uncomfortable to the patient, reaching in such a way a higher mean of injected volumes compared with the literature (Aliprandi et al., 2006)

In this study, direct intra-articular injection of contrast solution was applied to distend the joint capsule and to improve the assessment of small intra-articular structures such as the glenoid labrum using US guided anterior and posterior approaches as applied with (Choi et al., 2001; Jacobson et al., 2003; Magee et al., 2009) .

Most of these articles had shown superior sensitivity and accuracy of direct MR arthrography over conventional MR imaging, in particular for the assessment of the lesions of the glenoid labrum and partial articular surface tears of the rotator cuff muscles.

In the current study Bankart's lesion was the commonest abnormality. Lesion were present is 30 patients (60\% of the cases). These results are in accordance with previous reports which have demonstrated that Bankart's lesions are present in 60-90\% of shoulder dislocations (Horst et al. 2014; Jordan et al. 2015; Saders et al. 2010). Bankart's lesions were further subdivided into classic Bankart's $(n=10)$, bony Bankart's $(n=11)$ and Bankart's variants ( $\mathrm{n}=5$ ALPSA, 2 Perthe's and 1 GLAD). Classic and bony Bankart's lesions were the most frequent types of Bankart's encountered on the present study. In the current study, MRA showed sensitivity of about $90 \%$ for the detection of classic Bankart's (Figure 1). The agreement with arthroscopy for classic Bankart's was substantial $(\kappa=0.72)$. The $2^{\text {nd }}$ most frequently encountered type of Bankart's in the current work 
is the 'bony Bankart's lesion' (Figure 2). This

osseous defect along the
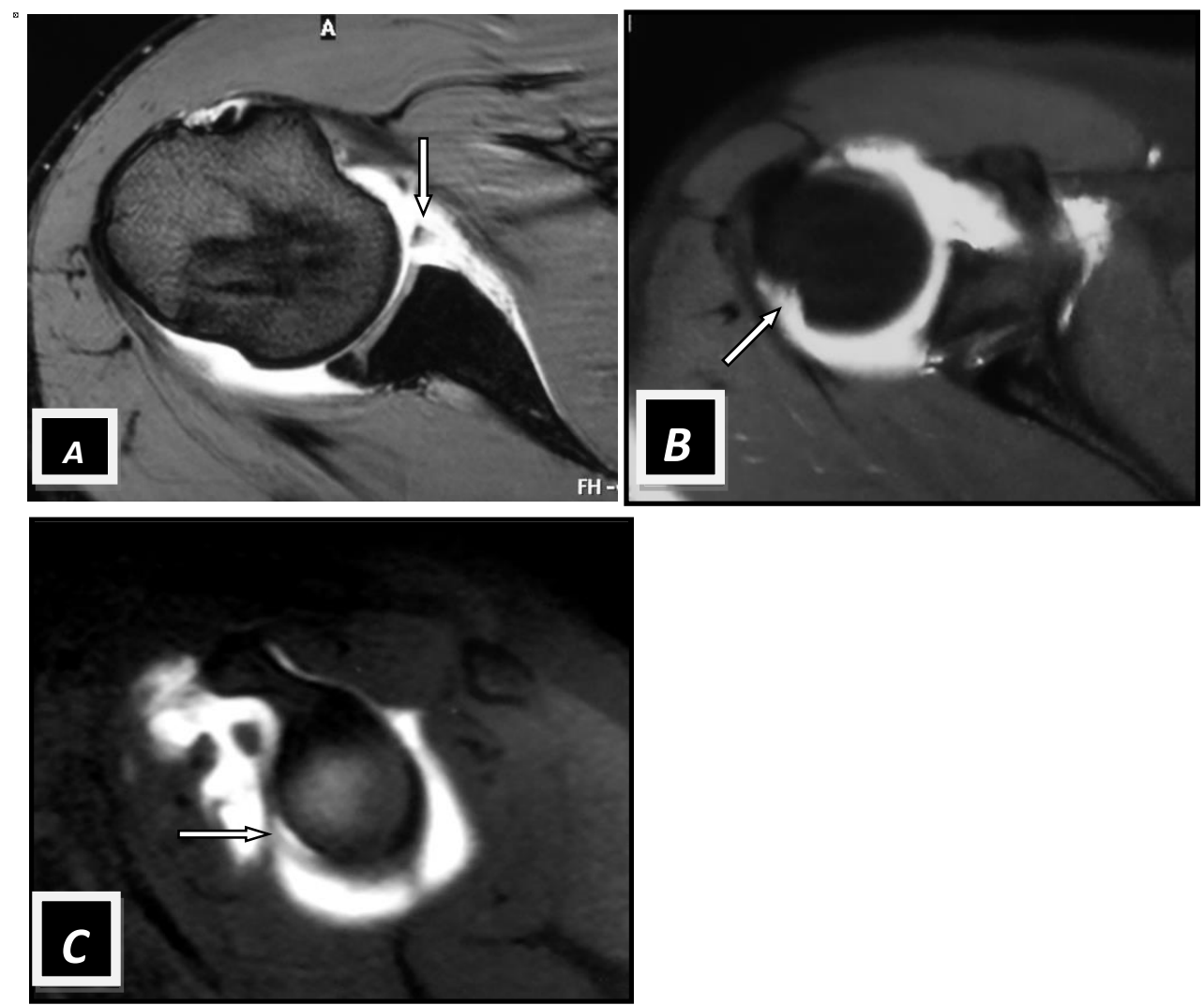

Fig. 1. Axial GR and T1WI with fat suppression (A, B) \& sagittal oblique (C) T1WI with fat suppression MRA of the right shoulder showed Hill Sachs lesion (arrow in B) and classic Bankart's lesion (arrows in A and $\mathrm{C})$.
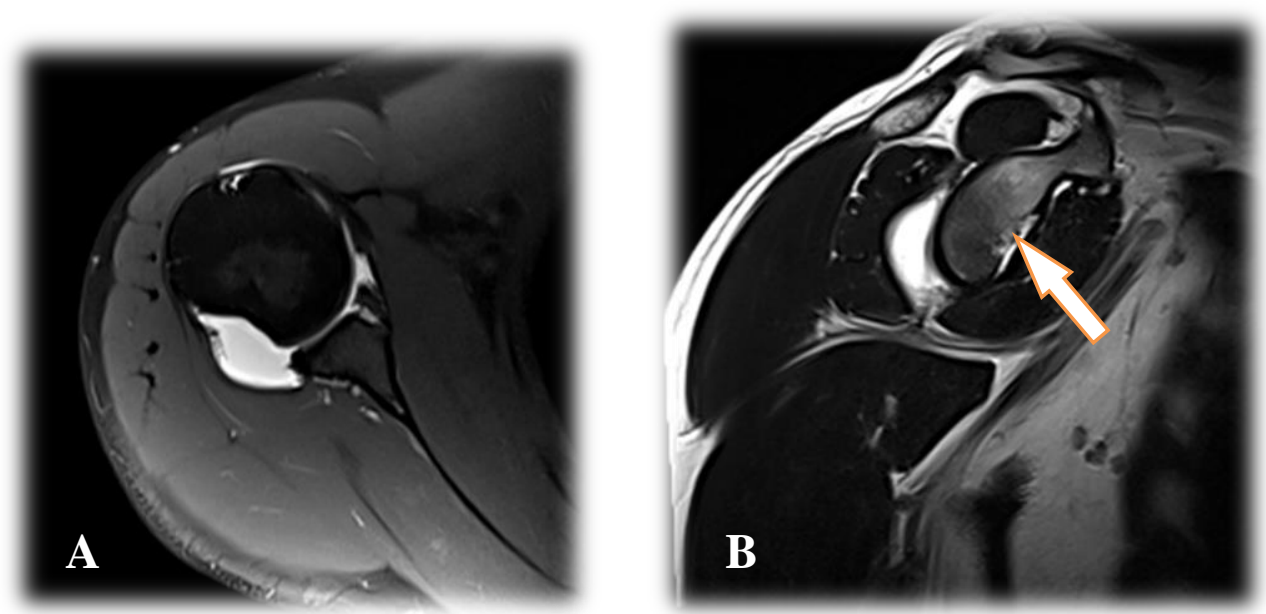

Fig. 2. Fat suppressed axial (A) \& sagittal T2 weighted (B) images $M R$ arthrogram showed contrast leak at the base of the detached anteroinferior labrum along with a detached glenoid bone fragment (arrows, Bony Bankart's). 
Anteroinferior margin of the glenoid is induced by the anterior and inferior translation and impaction of the head of the humerus against this glenoid rim. A variable sized bone fragment is avulsed together with the labro-ligamentous complex (Rabinowitz et al., 2017). In the present work, MRA showed sensitivity of 91.8, and specificity of $100 \%$, with substantial agreement with surgery $(\kappa=0.88)$ for bony Bankart lesions. These results disagree with (Jordan et al., 2015) which showed lower sensitivity in identifying osseous Bankart lesions using MRA (0.8) and suggested that CT scans should be considered if further clarification on osseous lesions is required.

Bankart's variants, such as Perthe's, ALPSA and GLAD lesions (Figure 3), were represented by a small number in the present study. Perthe's lesion (a variant of Bankart's lesion), known since, cannot often be diagnosed on conventional MRI, and sometimes, may be unnoticed during arthroscopy; According to (Waldt et al., 2004). Perthe's lesion sometimes can be difficult to assess on MR arthrography, especially in the presence of adhesions that prevent infiltration of intra-articular contrast medium. In such cases, the accuracy of MR arthrography can be increased by placing the patient's arm in ABER (Abduction and extrarotation of the arm) position during scan. The ABER position is thought to induce a tensile force on the anterior labro-ligamentous complex allowing subtle trickling of the injected contrast solution between the glenoid and pseudo-normal appearing labrum leading to increased likelihood for detection of such subtle or undisplaced anterior tears (Wischer et al. 2002).
Five ALPSA lesions were found at arthroscopy. These lesions are commonly found in patients with chronic anterior shoulder instability, with scar tissue formation and chronic changes in the labroligamentous complex. The criteria for diagnosis of ALPSA lesion on MRA examination were an intact medially stripped scapular periosteum along with the detached labroligamentous complex against labrum against the glenoid neck and the absence of normal labrum on the glenoid rim (De Coninck et al., 2016). In the current study, MRA nicely revealed the intact medially stripped anterior scapular periosteum together with the labro-ligamentous complex.

Impaction of the head of the humerus against the glenoid rim during shoulder dislocation may give rise to bony contusions and fractures. These lesions are located either at the posterosuperior margin of the humeral head in case of anterior shoulder dislocation and are known as Hill-Sachs lesions or at the anterior margin of the humeral head and are known as reverse HillSachs lesions (also called trough or McLaughlin's fractures) in case of posterior shoulder dislocation. A Hill-Sachs lesion (Figure 3) is a traumatic defect postero-superolateral aspect of the humeral head. It results from impaction of this part of the humeral head against the anterior-inferior portion of the glenoid rim during anterior dislocation. The typical location of Hill-Sachs lesion is at or above the level of the coracoid process to avoid confusion with the slight flattening of the postero-inferior surface of the humeral head; which could lead to false positive diagnosis of Hill Sach's fracture (Kadi et al., 2017). 

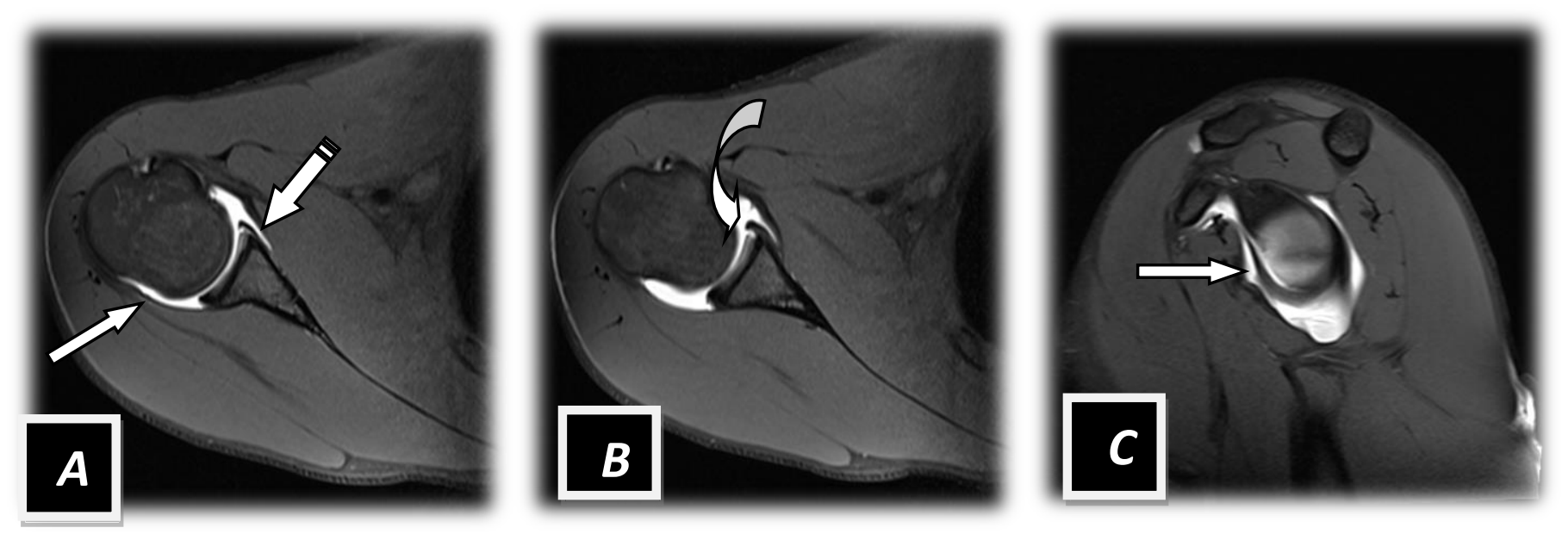

Fig.3. Axial (A and B) \& sagittal (C) T1WIs with fat suppression MR arthrogram showed Small Hill Sachs lesion (image A, arrow) detached non-displaced anterior and inferior labrum (images B and D) associated with full thickness chondral defect at the anterior glenoid (image C, curved arrow) consistent with GLAD lesion.

In the present study, Hill-Sachs fracture was seen in 26 patients with anterior instability. MRA missed the diagnosis of two cases confirmed by arthroscopy. The sensitivity and specificity of MRA for the detection of Hill-Sachs were $92.31 \%$ (24/26), $100 \%$ respectively and accuracy of $96.15 \%$, with almost perfect agreement with arthroscopy $(\kappa=0.92)$. Our results regarding Hill Sachs lesions are agreed by previous a previous study by (Oh et al., 2010) .

MR arthrography and MRI are generally considered superior to ultrasound imaging and conventional arthrography in the evaluation of RC disorders. At present, preoperative MRA or MRI might be needed for better evaluation of the rotator cuff condition, such as the condition of torn tendon and severity of fatty infiltration of muscles. Previous studies have compared the diagnostic performance of MRA to that of MR imaging and ultrasound imaging for the detection of rotator cuff tears (RCT). In a meta-analysis of the literature, (De Jesus et al., 2009) concluded that MR arthrography is superior to conventional MRI and ultrasound imaging for the diagnosis of full and partial-thickness RCTs (sensitivity of $91.7 \%$ and specificity of $96.5 \%$ when all tears were considered, sensitivity of $85.9 \%$ and specificity of $96 \%$, when partial-thickness tears were considered). Our results for MRA are in accordance to those in the previous reports. 
In the current work, arthroscopy confirmed a total number of 8 rotator cuff tears $(n=1$ full thickness and $n=7$ partial thickness tendon tears). For the detection of RCTs on the current study, full thickness tear is considered if it results in a connection between the two surfaces of the tendon with focal or diffuse contrast filled gap in the entire thickness of the tendon, and extension of intra-articular injected contrast solution through it into the subacromial-subdeltoid bursa (Lecouvet et al., 2008)

Partial-thickness tears, which don't involve the whole thickness of the tendon, were classified as bursal surface partial tear, intrasubstance partial tear, or articular surface partial tear depending on location.

Based on our results, MRA had substantial agreement $(\kappa=0.85)$ with arthroscopy in the detection of RCTs (sensitivity, specificity, predictive values, and accuracy) of MRA were $100 \%, 95.24 \%, 80 \%, 100 \%$ and $97.26 \%$ ). Regarding partial thickness rotator cuff tears, the current data, however, revealed that 7 cases were correctly diagnosed by MRA with the respective sensitivity and agreement with arthroscopy (77.7\% and $\kappa=0.85$ ).

Two cases of partial thickness RC tear were diagnosed at MRA but not depicted on arthroscopy (false positive result of MRA). These bursal sided partial thickness tears are theoretically visualized on arthroscopy using bursal approach. However, these superficial tears are often associated with subacromial sub deltoid bursitis, making their visualization on arthroscopy difficult as in our case (Fukuda, 2003) .

Superior labral antero-posterior (SLAP) lesions: are tears that involve the anchor of the long head of the biceps tendon (biceps labral complex). Since the initial description by Andrews et al. in 1985 (Andrews et a., 1985) and subsequent classification introduced by Snyder et al. (Snyder et al., 1990), SLAP lesions represent a diagnostic challenge for orthopaedic surgeons. SLAP lesions are difficult to be predicted on clinical examination resulting in a poor diagnostic value. Therefore, conventional magnetic resonance imaging (MRI) and MR arthrography (MRA) have become increasingly used in the workup of shoulder pain (Ahsan et al., 2016) .

Patients with SLAP lesions usually present with pain, clicking, and instability. A "peel-back" mechanism has been proposed where torsional loads across the superior labrum from the more posteriorly oriented bicep tendon displace the labrum and biceps tendon over the glenoid rim (Grossman et al., 2005).

SLAP tears in athletes performing repetitive overhead motions (such as swimmers) result in persistent pain are associated abnormalities of the rotator cuff. Four basic types of SLAP lesions were described. Over time, several other types have been described and added. At least 10 SLAP subtypes had been described until now. SLAP lesions range from simple fraying of the biceps labral complex through bucket-handle and flap tears to extension of tears into the biceps tendon and glenohumeral ligaments.

Sometimes, differentiation of SLAP lesions from anatomic variants of the superior labrum may be not possible. The orientation, thickness, and shape of the contrast line are key findings to help differentiae a tear from an anatomic variant (De Filippo et al., 2020). In the 
current study, SLAP lesions were the second most common labral abnormality after Bankart lesions and were confirmed by arthroscopy in 12 patients, of them MRA detected 11 SLAP lesions. The most common type of SLAP lesion in the present study was type II (Figure 4) and its variants, which is in accordance with the results of previous studies done by (Bencardino et al., 2000; Kim et al., 2011) (30,31).

We agree with (Magee et al., 2004; MohanaBorges et al., 2003; Sheridan et al., 2014) (32-34) when stating that MRA is the best imaging modality for the evaluation of SLAP lesions and capsular ligaments. MRA had a high diagnostic performance for detection of SLAP lesions with almost perfect agreement with arthroscopic findings $(\kappa=0.94)$ and showed sensitivity, specificity and positive predictive value of $91.6 \%$, $100 \%$ and $97.4 \%$ and accuracy of $92 \%$ for the detection of SLAP lesions.

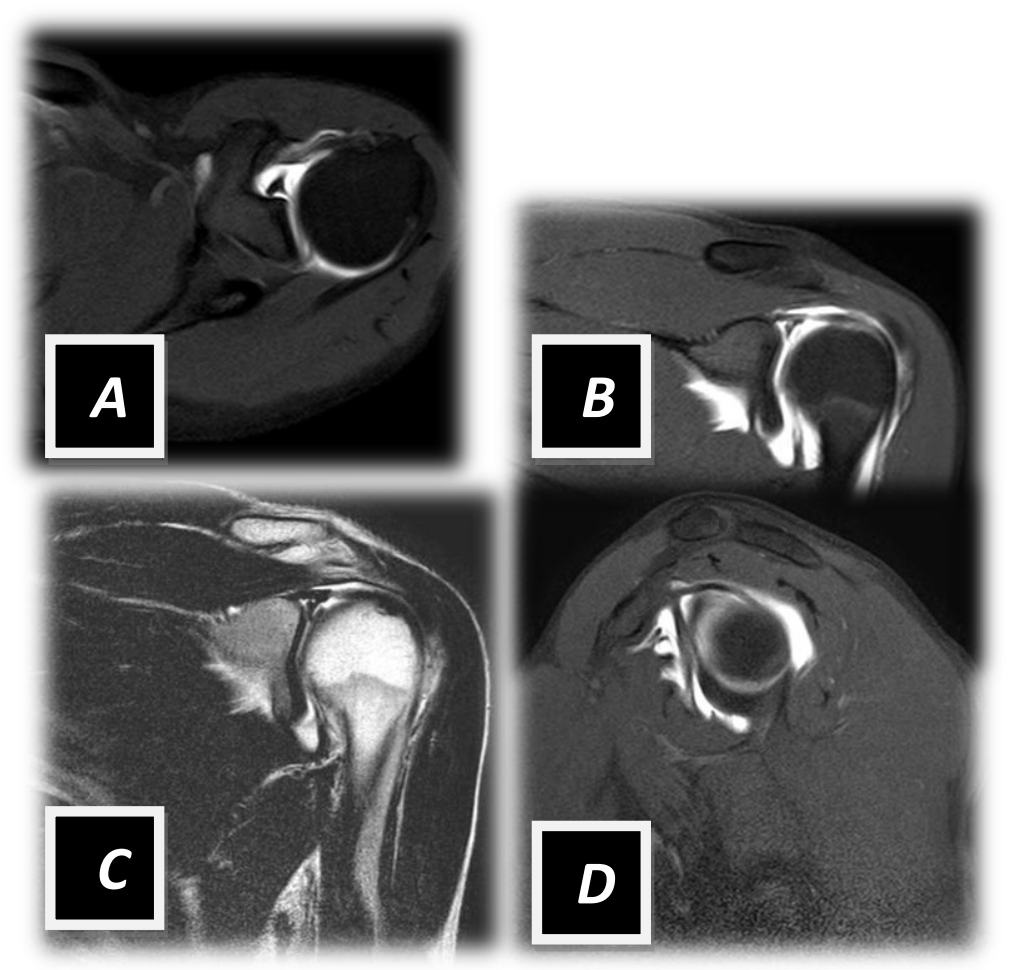

Fig. (4): Axial (A), coronal oblique (B), and sagittal T1 (D) weighted fat suppressed and coronal T2WI (C) MR arthrogram showed stripping of the superior labrum and biceps tendon from the glenoid (SLAP type IIA lesion).

\section{Conclusion:}

Glenohumeral instability remains a very complex and sometimes challenging diagnostic problem.
There are potential lesions of the capsule and labro-ligamentous structures that can occur in association with shoulder instability, and the 
clinical history and physical findings are not always sufficient to make accurate diagnosis.

Reliable diagnostic imaging is mandatory not only for establishing a correct diagnosis but also for evaluation when different treatment options are considered. Imaging is especially important when clinical findings are contradictory or sparse. In the past, several imaging modalities were used ranging from conventional radiography, arthrography to computerized axial arthrography. Recently, various imaging modalities are now available including ultrasonography, CT arthrography, conventional MRI and MR arthrography. Magnetic resonance imaging is an excellent method for assessment of disorders of the shoulder. Conventional MR is sensitive and specific for detection of rotator cuff pathology but MR arthrography is necessary to attain high sensitivity and specificity for detection of labral pathology.

The choice of using either conventional MR or MR arthrography is usually made based on the clinical history. If there is a history suggesting is instability, MR arthrography will be often performed, while, if the clinical suggests rotator cuff pathology, conventional MR will be usually performed. The drawback to this is that clinical examination of the shoulder is inaccurate when compared with arthroscopy.

If the choice of whether to perform MR arthrography or not is based only on the clinical findings, there will be a number of patients with labral pathology who will present with vague or non-specific symptoms and may be imaged using conventional MRI. Because conventional MR does not have a high diagnostic accuracy for the detection of labral lesions, a labral tear could be missed in. Unfortunately, however, arthrography is a time-consuming, minimally invasive technique, and although generally safe, it could carry risk to the patient.

It is not all reasonable to perform MR arthrography on all patients. Therefore, certain criteria are needed to determine which group of patients would benefit most from MR arthrography. (Magee et al., 2004) showed that certain groups of professional athletes benefit from the use of routine MR arthrography, but this is a very specific patient cohort. Patient age can be as a discriminator where patients 40 years of age and less are routinely imaged with MR shoulder arthrography, whereas patients older than 40 years of age are routinely imaged with conventional MRI (Performing arthrography in the latter age group would be for only those who have high clinical suspicion of labral pathology and in whom presence of such a tear would change the clinical management).

In our study, MR arthrography showed labral tears with greater sensitivity than conventional MRI in addition to what it afforded in imaging capsuloligamentous, cartilaginous injuries as well as small partial or complete tears of the rotator cuff tendons.

In the present study, the sensitivity of MR arthrography was $78 \%$, and $100 \%$ specificity. Positive predictive value between MR arthrogram and arthroscope was $100 \%$ and negative predictive value was $21 \%$ 
In conclusion, our results recommend that MR arthrography should be increasingly used, especially when there is a high possibility of labral or capsular tear on clinical examination and also to assess the joint capsule itself, and the extent of the labro-ligamentous injury.

\section{Conflict of Interest:}

The authors of the study have no conflict of interest related to this publication.

\section{References:}

1. Chang I-YJ, Polster JM (2016). Pathomechanics and Magnetic Resonance Imaging of the Thrower's Shoulder. Radiologic Clinics of North America, 54 (5): 801-815.

2. Degen RM, Giles JW, Thompson SR, Litchfield RB, Athwal GS (2013). Biomechanics of Complex Shoulder Instability. Clinics in Sports Medicine, 32 (4): 625-636.

3. Abboud JA, Soslowsky LJ (2002). Interplay of the static and dynamic restraints in glenohumeral instability. Clinical Orthopaedics and Related Research, 400(5): 48-57.

4. Omoumi P, Mercier GA, Lecouvet F, Simoni P, Vande Berg BC (2010). CT arthrography, MR arthrography, PET, and scintigraphy in osteoarthritis. Radiologic Clinics of North America, 47(4): 595-615.

5. Chang D, Mohana-Borges A, Borso M, Chung CB (2008). SLAP lesions: anatomy, clinical presentation, MR imaging diagnosis and characterization. Eur J Radiol, 68(1):7287.

6. Chundru U, Riley GM, Steinbach LS (2009). Magnetic Resonance Arthrography.
Radiologic Clinics of North America, 47(3): 471-494.

7. Burke CJ, Rodrigues TC, Gyftopoulos S (2020). Anterior Instability. Magnetic Resonance Imaging Clinics of North America, 28(2): 195-209.

8. Sconfienza LM, Albano D, Messina C, Silvestri E, Tagliafico AS (2018). How, when, why in magnetic resonance arthrography: an International Survey by the European Society of Musculoskeletal Radiology (ESSR). Eur Radiol, 28 (23): 5668.

9. Gokalp G, Dusak A, Yazici Z (2010). Efficacy of ultrasonography-guided shoulder MR arthrography using a posterior approach. Skeletal Radiology, 39(6): 575579.

10. Aliprandi A, Fausto A, Quarenghi M, Modestino S, Randelli P, Sardanelli F (2006). One-shot CT and MR arthrography of the shoulder with a mixture of iodinated and paramagnetic contrast agents using arthroscopy as a gold standard. La radiologia medica, 111(1): 53-60.

11. Choi JA, Suh S, Kim BH, Cha SH, Kim M G, Lee KY, et al. (2001). Comparison between Conventional MR Arthrograhphy and Abduction and External Rotation MR Arthrography in Revealing Tears of the Antero-Inferior Glenoid Labrum. Korean Journal of Radiology, 2(4): 216-227.

12. Jacobson JA, Lin J, Jamadar DA, Hayes C W (2003). Aids to Successful Shoulder Arthrography Performed with a Fluoroscopically Guided Anterior Approach 1. Radiographics, 23(2): 373-378.

13. Magee, T. (2009). 3-T MRI of the Shoulder: Is MR Arthrography Necessary? American Journal of Roentgenology, 192(1): 86-92. 
14. Horst K, Von Harten R, Weber C, Andruszkow H, Pfeifer R, Dienstknecht T, et al (2014). Assessment of coincidence and defect sizes in Bankart and Hill-Sachs lesions after anterior shoulder dislocation: a radiological study. The British Journal of Radiology, 87(1034), 20130673.

15. Jordan RW, Naeem $\mathbf{R}$, Srinivas $\mathbf{K}$, Shyamalan GA (2015). A comparison of magnetic resonance arthrography and arthroscopic findings in the assessment of anterior shoulder dislocations. Skeletal Radiology, 44(5): 653-657.

16. Sanders TG, Tirman P F, Linares R, Feller JF, Richardson $R$ (2010). The glenolabral articular disruption lesion: MR arthrography with arthroscopic correlation. American Journal of Roentgenology, 172(1): 171-175.

17. Rabinowitz J, Friedman R, Eichinger JK (2017). Management of Glenoid Bone Loss with Anterior Shoulder Instability: Indications and Outcomes. Current Reviews in Musculoskeletal Medicine, 10(4): 452-462

18. Jordan RW, Saithna A (2015). Physical examination tests and imaging studies based on arthroscopic assessment of the long head of biceps tendon are invalid. Knee Surgery, Sports Traumatology, Arthroscopy, 25(10): 3229-3236.

19. Waldt S, Burkart A, Lange P, Imhoff AB, Rummeny EJ, Read KW (2004). Diagnostic performance of MR arthrography in the assessment of superior labral anteroposterior lesions of the shoulder. American Journal of Roentgenology, 182(5): 1271-1278.

20. Wischer TK, Bredella MA, Genant HK, Stoller DW, Bost FW, Tirman PF (2002). Perthes Lesion (A Variant of the Bankart Lesion) MR Imaging and MR Arthrographic
Findings with Surgical Correlation. American Journal of Roentgenology, 178(1): 233-237.

21. De Coninck T, Ngai SS, Tafur M, Chung CB (2016). Imaging the Glenoid Labrum and Labral Tears. RadioGraphics, 36(6): 16281647.

22. Kadi R, Milants A, Shahabpour M (2017). Shoulder Anatomy and Normal Variants. Journal of the Belgian Society of Radiology, 101(S2), 3. doi:10.5334/jbrbtr. 1467

23. Oh JH, Kim JY, Choi JA, Kim WS (2010). Effectiveness of multidetector computed tomography arthrography for the diagnosis of shoulder pathology: Comparison with magnetic resonance imaging with arthroscopic correlation. Journal of Shoulder and Elbow Surgery, 19(1): 14-20.

24. De Jesus, JO, Parker L, Frangos AJ, Nazarian LN (2009). Accuracy of MRI, MR arthrography, and ultrasound in the diagnosis of rotator cuff tears: a meta-analysis. American Journal of Roentgenology, 192(6): 1701-1707.

25. Lecouvet FE, Simoni $\mathbf{P}$, Koutaïssoff $\mathbf{S}$, Vande Berg BC, Malghem J, Dubuc JE (2008). Multidetector spiral CT arthrography of the shoulder. European Journal of Radiology, 68(1): 120-136.

26. Fukuda H (2003). The management of partialthickness tears of the rotator cuff. Journal of Bone And Joint Surgery-British Volume, 85(1): 3-11

27. Ahsan ZS, Hsu JE, Gee AO (2016). The Snyder Classification of Superior Labrum Anterior and Posterior (SLAP) Lesions. Clinical Orthopaedics and Related Research®, 474(9): 2075-2078.

28. Grossman MG, Tibone JE, McGarry MH, Schneider DJ, Veneziani S, Lee TQ (2005). A Cadaveric Model of the Throwing 
Shoulder. The Journal of Bone \& Joint Surgery, 87(4): 824-831.

29. De Filippo M, Schirò S, Sarohia D, Barile A, Saba L, Cella S, et al. (2020). Imaging of shoulder instability Skeletal Radiol. Oct; 49(10):1505-1523.

30. Bencardino JT, Beltran J, Rosenberg ZS, Rokito A, Schmahmann S, Mota J, et al. (2000). Superior labrum anterior-posterior lesions: diagnosis with MR arthrography of the shoulder. Radiology, 214(1): 267-271.

31. Kim TK, Queale WS, Cosgarea AJ, McFarland EG (2011). Clinical features of the different types of SLAP lesions: an analysis of one hundred and thirty-nine cases. J Bone Joint Surg Am, 85 (2):66-71.

32. Magee T, Williams D, Mani N. (2004). Shoulder MR arthrography: which patient group benefits most? AJR Am J Roentgenol,183(4): 969-974

33. Mohana-Borges AV, Chung CB, Resnick D (2003). Superior labral anteroposterior tear: classification and diagnosis on MRI and MR arthrography.American Journal of Roentgenology, 181(6): 1449-1462.

34. Sheridan K, Kreulen C, Kim S, Mak W, Lewis K, Marder $R$ (2014). Accuracy of magnetic resonance imaging to diagnose superior labrum anterior-posterior tears. Knee Surgery, Sports Traumatology, Arthroscopy, 23(9): 2645-2650. 